\title{
Cytosolic galectin-7 impairs p53 functions and induces chemoresistance in breast cancer cells
}

\author{
Andrée-Anne Grosset ${ }^{1,2}$, Marilyne Labrie ${ }^{1}$, Donald Gagné ${ }^{1}$ Maria-Claudia Vladoiu', Louis Gaboury², \\ Nicolas Doucet ${ }^{1}$ and Yves St-Pierre $^{1 *}$
}

\begin{abstract}
Background: Resistance to apoptosis induced by anti-cancer drugs is a major obstacle for the treatment of aggressive forms of breast cancer. Galectin-7 (gal-7) was recently shown to be specifically expressed in basal-like but not in luminal subtypes of human breast cancer.

Methods: We generated a mutant form of gal-7 (R74S). Arginine 74 is the structural equivalent of arginine 186 found in human galectin-3. Mutation R1865 was previously shown to abolish the biological function of galectin-3.

Results: Mutation of arginine 74 induced only limited and local changes to the gal-7 fold. Recombinant forms of R74S and wtgal-7 were also equally effective at forming dimers in solution. Analysis of the thermodynamic parameters by isothermal titration calorimetry (ITC) indicated, however, that binding of lactose to gal-7 was inhibited by the R74S mutation. Using confocal microscopy and electron microscopy, we confirmed the expression of gal-7 in the cytosolic and nuclear compartments of breast cancer cells and the ability of gal-7 to translocate to mitochondria. The mutation at position 74, however, greatly reduced the expression of gal-7 in the nuclear and mitochondrial compartments. Interestingly, cells expressing mutated gal-7 were equally if not even more resistant to drug-induced apoptosis when compared to cells expressing wtgal-7. We also found that both wtgal-7 and R74S inhibited dox-induced PARP-1 cleavage and p53 protein expression. The inhibition of p53 correlated with a decrease in p21 protein expression and CDKN1A mRNA. Furthermore, analysis of nuclear and cytoplasmic fractions showed that both wild type and R74S mutant gal-7 inhibited p53 nuclear translocation, possibly by increasing degradation of cytosolic p53.
\end{abstract}

Conclusions: These findings pose a challenge to the paradigm that has guided the design of galectin-specific inhibitors for the treatment of cancer. This study suggests that targeting CRD-independent cytosolic gal-7 in breast cancer cells may be a valuable strategy for the treatment of this disease. Our study will thus complement efforts towards improving selectivity of targeted anticancer agents.

Keywords: Galectin-7, Localization, Apoptosis, p53, Breast cancer

\section{Background}

Members of the galectin family are characterized by their ability to bind $\beta$-galactosides via a highly conserved carbohydrate recognition domain (CRD). They play an important role in several physiological processes, including embryonic development, intercellular adhesion, host-pathogen interactions, cell migration, and immune response [1]. They are normally classified according to their structural organization. Galectins containing

\footnotetext{
* Correspondence: yves.st-pierre@iaf.inrs.ca

'INRS-Institut Armand-Frappier, 531 Blv. des Prairies, Laval, Quebec H7V 1B7, Canada

Full list of author information is available at the end of the article
}

only one CRD are called prototype and include galectins $1,2,5,7,10,11,13,14$ and 15 . Those with two distinct CRDs in tandem connected by a linker region (tandemrepeat type) are galectins 4, 6, 8, 9 and 12. Galectin-3 is the only member of the third group and is a chimeratype protein with one CRD connected to an unusual nonlectin domain rich in proline and glycine.

Historically, galectins have been known as small extracellular soluble that bind cell surface glycans, helping organizing membrane domains and regulating the signaling threshold and the receptor residency time [2]. Galectins, however, exhibits a wide range of subcellular 
localizations, being found in both intracellular and extracellular compartments. Intracellularly, they have been reported to be exclusively/predominantly cytosolic, nuclear, mitochondrial, or distributed between the distinct subcellular compartments. Finally, even within a specific organelle, they appear to be distributed diffusely or to form aggregates or punctate structures. Such wide subcellular distribution significantly complicate galectin-targeted anticancer therapy since the pro- and the anti-tumoral functions of galectins differ according their subcellular localization [3].

Galectin-7 (gal-7) is a prototype galectin that forms homodimers [4]. Gal-7 is preferentially expressed in stratified epithelia, including epidermis, cornea, oral cavity, esophagus and rectal epithelium [5]. It is also expressed in mammary myoepithelial cells in tissues of normal individuals [6]. Its level of expression, however, is significantly altered in various types of cancer [7]. For example, gal-7 is expressed at higher levels in aggressive molecular subtypes of breast carcinoma, most notably in basal-like breast cancer with an ER/PR/HER-2 negative status [6]. Exogenous expression of gal-7 in breast cancer cell lines that express low or undetectable levels of gal-7 resulted in an increased metastatic behavior to the lung and bone and larger osteolytic lesions. Such pro-tumoral function of gal-7 has been largely attributed to its ability to protect cancer cells from pro-apoptotic signals $[6,8]$. Like other galectins, however, gal-7 is preferentially expressed intracellularly, most notably in cytosolic, nuclear and mitochondrial compartments [9-12]. Whether the resistance of breast cancer cells to apoptosis is dependent on the intracellular localization of gal-7 remains unknown. In the present work, we have addressed this question by generating a mutant form of gal-7 (R74S) with altered subcellular localization and tested its ability to mediate resistance of breast cancer cells to druginduced cell death.

\section{Methods}

\section{Tissue microarrays and immunohistochemistry}

Representative specimens from our previous TMA analysis were immunostained for gal-7 using the Discovery XT automated immunostainer (Ventana Medical Systems, Tucson, AZ) [6]. Deparaffinized sections were incubated in Cell Conditioning 1 (pH 8.0) for antigen retrieval and then stained for 60 min with the anti-human gal-7 polyclonal antibody (R\&D Systems, Minneapolis, MN) using a 1:150 dilution. The slides were counterstained with hematoxylin and bicarbonate. Each section was scanned at a high resolution using the Nanozoomer Digital Pathology System (Hamamatsu, Bridgewater, NJ). The study was approved by the research ethics committee of the research center at the Centre Hospitalier de l'Université de Montréal (approval No. SL 05.019).

\section{Cell lines and reagents}

The MCF-7 and MDA-MB-468 cell lines were provided by Dr. Peter Siegel (Rosalind and Morris Goodman Cancer Research Centre, McGill University, Montreal, QC, Canada) and maintained in Dulbecco's Modified Eagle's Medium (DMEM) supplemented with 10\% (v/v) FBS, $2 \mathrm{mM}$ L-glutamine, $10 \mathrm{mM}$ HEPES buffer and $1 \mathrm{mM}$ sodium pyruvate. SKBR3 cells, obtained from Dr. Sylvie Mader (Institute for Research in Immunology and Cancer, University of Montreal, Montreal, QC, Canada), were grown in McCoy's 5A Medium supplemented with 10\% (v/v) FBS, $2 \mathrm{mM} \mathrm{L}$-glutamine and $10 \mathrm{mM}$ HEPES buffer at $37^{\circ} \mathrm{C}$ in a humidified atmosphere containing $5 \% \mathrm{CO}_{2}$. MCF10A and MCF12A protein extracts were provided by Dr. Isabelle Plante (INRS-Institut Armand-Frappier, Laval, QC, Canada). All cell culture products were purchased from Life Technologies (Burlington, ON, Canada). Cobalt chloride and lactose were purchased from Fisher Scientific (Ottawa, ON, Canada). MG-132 was from Cayman Chemical (Ann Arbor, MI). All other reagents were purchased from Sigma-Aldrich (St. Louis, MO), unless otherwise indicated.

\section{Generation of stable transfectants expressing gal-7 and gal-7 R74S}

To obtain stable MCF-7 breast carcinoma transfectants expressing gal-7, the cDNA encoding the human gal-7 (provided by Dr. Thierry Magnaldo) was cloned in $\mathrm{sr} \alpha$ eukaryotic expression vector (kind gift of Dr. François Denis) using SpeI and BamHI restriction enzymes. The replacement of arginine 74 to serine (R74S) was introduced by oligo-directed site-specific mutagenesis using the forward (5'-GGC CGC GAG GAG TCC GGG CCG GGC GTT CCT- $3^{\prime}$ ) and reverse (5' -GGC CGC GAG GAG TCC GGG CCG GGC GTT CCT- 3') primers. Controls were generated using MCF-7 breast carcinoma cells transfected with the empty sra vector. Transfection was carried out using Lipofectamine 2000 according to the manufacturer's instructions (Life Technologies). After $48 \mathrm{~h}$ of culture, transfected cells were allowed to grow in complete medium containing $1 \mu \mathrm{g} / \mathrm{ml}$ of puromycin. Individual colonies were expanded and gal-7 expression was monitored by Western blot analysis. All experiments were conducted with at least two independent clones expressing either wild type or mutant gal-7.

\section{RNA isolation ant RT-PCR}

Total cellular RNA was isolated from cells using the TRIzol reagent (Life Technologies) according to the manufacturer's instructions. First-strand cDNA was prepared from $2 \mu \mathrm{g}$ of cellular RNA in a total reaction volume of $20 \mu \mathrm{L}$ using the reverse transcriptase Omniscript (QIAGEN, Mississauga, ON, Canada). After reverse transcription, human p53 (gene ID 7157, sense primer: 5'- CCA 
GCC AAA GAA GAA ACC A -3' and antisense primer: 5' - TAT GGC GGG AGG TAG ACT GA -3'), human $p 21$ (gene ID 1026, sense primer: 5'- CTG GAG ACT CTC AGG GTC GAA -3' and antisense primer: 5' - GGA TTA GGG CTT CCT CTT GGA -3') and GAPDH (gene ID 2597, sense primer: 5' - CGG AGT CAA CGG ATT TGG TCG TAT-3' and antisense primer: 5'-CAG AAG TGG TGG TAC CTC TTC CGA -3') cDNAs were amplified using the following conditions: $94^{\circ} \mathrm{C}$ for $3 \mathrm{~min}$, followed by 25 to 35 cycles of the following: $94^{\circ} \mathrm{C}$ for 40 seconds, $60^{\circ} \mathrm{C}$ for 40 seconds, and $72^{\circ} \mathrm{C}$ for 40 seconds, followed by a final extension step at $72^{\circ} \mathrm{C}$ for $10 \mathrm{~min}$. PCR was performed in a thermal cycler (Eppendorf, Mississauga, ON, Canada). The amplified products were analyzed by electrophoresis using 1.5\% agarose gels and SYBR Safe (Life Technologies) staining and UV illumination.

\section{Co-immunoprecipitation}

MCF-7 stable transfectants expressing exogenous gal-7 and R74S mutant and MCF10A were transfected with vectors encoding wild type p53 (Origene, Burlington, MA). After 24 hrs, the cells were lysed in immunoprecipitation (IP) buffer containing 2\% (v/v) CHAPS, $50 \mathrm{mM}$ Tris pH 7.5, $150 \mathrm{mM} \mathrm{NaCl}, 0.1 \mathrm{mM}$ EDTA and protease inhibitors (Roche, Laval, QC, Canada). Equal amounts of whole cell protein extracts were used for each IP. Rabbit anti-p53 antibody (FL393; Santa Cruz Biotechnology, Santa Cruz, CA) or IgG control antibody $(2 \mu \mathrm{g})$ were incubated $10 \mathrm{~min}$ at room temperature with Dynabeads Protein G (Life Technologies). The Dynabeads-antibody complex was incubated with proteins overnight at $4^{\circ} \mathrm{C}$. After several washes in IP buffer, the protein complexes were resuspended in Laemmli loading buffer. Immunoprecipitated proteins were separated on a 15\% SDS-PAGE gel and analyzed by Western blotting using anti-gal-7 and anti-p53 as described below.

\section{Western blot analysis}

Whole cell extracts were suspended using RIPA lysis buffer (Thermo Fisher Scientific, Rockford, IL) and protease inhibitors (Roche). Mitochondria and nuclear proteins were extracted using a kit (Thermo Fisher Scientific; Sigma-Aldrich) following the manufacturer's instructions. Protein concentrations were measured using a protein assay reagent (Bio-Rad Laboratories, Mississauga, ON, Canada). Equal amounts of proteins were separated on SDS-PAGE and transferred onto nitrocellulose membranes (Bio-Rad Laboratories). The membranes were first blocked with 5\% (v/v) milk in PBS/0.05\% Tween 20 for 1 $\mathrm{h}$ and subsequently blotted overnight at $4^{\circ} \mathrm{C}$ with primary antibodies: goat anti-human gal-7 polyclonal antibody (1:1000; R\&D Systems, Minneapolis, MN), rabbit antip53 (FL393; 1:1000; Santa Cruz Biotechnology), rabbit anti-p21 (1:1000; Cell Signaling Technology, Danvers, $\mathrm{MA})$, rabbit anti-poly (ADP-ribose) polymerase (PARP)-1 (p25) monoclonal antibody (1:10000; Epitomics, Burlingame, CA), rabbit anti-COX IV polyclonal antibody (1:1000; Cell Signaling Technology), mouse anti-lamin $\mathrm{A} / \mathrm{C}$ monoclonal antibody (1:1000; Cell Signaling Technology), rabbit anti- $\beta$ tubulin monoclonal antibody (1:10000; Cell Signaling Technology), and mouse anti- $\beta$-actin monoclonal antibody (1:20000; Sigma-Aldrich). Secondary antibodies consisted of horseradish peroxidase conjugated donkey anti-goat (R\&D Systems), anti-rabbit or anti-mouse (GE Healthcare, Buckinghamshire, England). Detection was performed by the enhanced chemiluminescence method (GE Healthcare).

\section{Immuno-electron microscopy}

Cells were fixed in a $0.1 \%(\mathrm{v} / \mathrm{v})$ gluteraldehyde and $4 \%$ $(\mathrm{v} / \mathrm{v})$ paraformaldehyde solution and embedded in the low viscosity embedding Spurr media. Ultrathin sections were cut, placed on nickel grids and incubated in sodium metaperiodate. Samples were blocked in $1 \%(\mathrm{v} / \mathrm{v})$ BSA for $5 \mathrm{~min}$, incubated $60 \mathrm{~min}$ in a goat anti-human gal-7 polyclonal antibody (1:150) and $60 \mathrm{~min}$ in a rabbit anti-goat $10 \mathrm{~nm}$ gold-conjugated secondary antibody (1:20, Electron Microscopy Sciences, Hatfield, PA). Each section were counterstained with uranyle acetate and lead citrate and visualized using a Hitachi 7100 transmission electron microscope.

\section{Apoptosis detection by flow cytometry}

The percentage of apoptotic cells was measured by twocolor flow cytometry using Alexa Fluor 488 annexin V conjugate (Life Technologies) and propidium iodide (PI). Briefly, $1.75 \times 10^{5}$ cells were treated with $150 \mu \mathrm{M}$ cobalt chloride overnight at $37^{\circ} \mathrm{C}$ without serum. Cells were then harvested, stained and analyzed by flow cytometry using a FacsCalibur (BD Biosciences, San Jose, CA).

\section{Production of recombinant gal-7}

Each of the DNA fragments coding for gal-7 and R74S was cloned into pET-22b(+) using NdeI and HindIII restriction enzymes. Recombinant proteins were expressed in E. coli BL21(DE3) at $37^{\circ} \mathrm{C}$ following addition of $1 \mathrm{mM}$ IPTG at an $\mathrm{OD}_{600 \mathrm{~nm}}=0.6-0.7$ and an incubation of 4 h. Bacterial pellets were resuspended in lysis buffer $(0.7 \mathrm{mg} / \mathrm{mL}$ lysozyme, $10 \mathrm{mM}$ Tris $\mathrm{pH} 8,100 \mathrm{mM}$ $\mathrm{NaCl}, 1 \mathrm{mM}$ EDTA, $1 \mathrm{mM}$ DTT and protease inhibitor cocktail), incubated for $1 \mathrm{~h}$ at $37^{\circ} \mathrm{C}$ and centrifuged for $30 \mathrm{~min}$ at $15000 \mathrm{~g}\left(4^{\circ} \mathrm{C}\right)$. The supernatant was then filtered and applied to a lactose-agarose column and the protein was eluted in one $\mathrm{mL}$ fractions with 150 $\mathrm{mM}$ lactose. Fractions were analyzed by SDS-PAGE. Gal-7 and R74S were dialyzed against $20 \mathrm{mM}$ potassium phosphate at $\mathrm{pH} 7.2$ for all subsequent characterization experiments. ${ }^{15} \mathrm{~N}$-labeled samples were prepared by 
growing E. coli BL21(DE3) in M9 minimal medium as previously described [13].

\section{Solution NMR experiments}

Proteins were at a concentration of $535 \mu \mathrm{M}$ for gal-7 and $200 \mu \mathrm{M}$ for gal-7 R74S. A 10\% (v/v) $\mathrm{D}_{2} \mathrm{O}$ solution was added to the protein samples for NMR spin-lock purposes. Protein concentration was determined by UV-vis spectrophotometry using an extinction coefficient of 8030 $\mathrm{M}^{-1} \mathrm{~cm}^{-1}$ at $280 \mathrm{~nm}$ [14]. ${ }^{1} \mathrm{H}_{-}{ }^{15} \mathrm{~N}$ HSQC spectra were acquired at $800 \mathrm{MHz}$ on a Varian (Agilent) NMR spectrometer equipped with a triple resonance probe and pulsed-field gradients. All spectra were acquired at $310 \mathrm{~K}$ as calibrated with a standard methanol sample. The ${ }^{1} \mathrm{H}-{ }^{15} \mathrm{~N}$ HSQC experiments were conducted with $256 t_{1}$ and 8192 $t_{2}$ points with proton and nitrogen spectral widths of 3000 and $8000 \mathrm{~Hz}$, respectively. Spectra were processed using NMRPipe [15] and further analysed using Sparky [16]. The ${ }^{1} \mathrm{H}-{ }^{15} \mathrm{~N}$ composite chemical shift differences $(\Delta \delta)$ were calculated between wild-type and mutant enzymes according to the following equation [17]: $\Delta \delta$ (ppm) = $\left[\left(\Delta \delta_{\mathrm{HN}}^{2}+\Delta \delta_{\mathrm{N}}^{2} / 25\right) / 2\right]^{1 / 2}$. Only chemical shift variations showing $\Delta \delta>0.02 \mathrm{ppm}$ were considered significant.

\section{Isothermal titration calorimetry (ITC)}

Lactose was reconstituted in a $20 \mathrm{mM}$ potassium phosphate buffer at pH 7.2. Gal-7 and R74S were dialyzed in the same buffer after purification. All experiments were performed in a Nano ITC microcalorimeter (TA Instruments, New Castle, DE) at $25^{\circ} \mathrm{C}$ with a stirring rate of $250 \mathrm{rpm}$. Pre-equilibrated solutions of $200 \mu \mathrm{M}$ protein and $6 \mathrm{mM}$ ligand were used for each assay. A control experiment was performed by titrating lactose into proteinfree buffer. Each experiment consisted of 20 injections of $2 \mu \mathrm{L}$ ligand into protein, with an interval of 130 seconds between injections. All experiments were performed at least in triplicate. Data was analyzed and fitted using the NanoAnalyze software v2.3.6 (TA Instruments).

\section{FITC conjugation and gal-7 binding assay}

Briefly, $10 \mu \mathrm{l}$ of a $2 \mathrm{mg} / \mathrm{ml}$ fluorescein isothiocyanate (FITC)/DMSO solution was added to $300 \mu \mathrm{l}$ of $1.7 \mu \mathrm{g} / \mu \mathrm{l}$ recombinant wtgal-7 or R74S in a $0.1 \mathrm{M} \mathrm{NaHCO} 3 \mathrm{pH}$ 9.2 solution and incubated for $2 \mathrm{hrs}$ at room temperature on a roller. FITC-conjugated wtgal-7 or R74S was then purified using a PD-10 sepharose column (GE healthcare) and eluted with PBS containing $0.01 \%$ [v/v] sodium azide (PBA). To measure FITC-wtgal-7 or R74S binding to cell surface, $2.5 \times 10^{5}$ cells were incubated for $30 \mathrm{~min}$ with the indicated concentrations. Cells were washed 2 times with PBA and resuspended in $500 \mu \mathrm{l}$ PBA. Samples were analyzed by FACSCalibur (BD Biosciences).

\section{Results}

Subcellular localization of gal-7 in human breast cancer cells

Analysis of gal-7 expression in normal mammary epithelium shows positive gal-7 staining in both the nuclear and cytoplasmic compartments of myoepithelial cells (Figure 1A). A similar pattern of expression was observed in tissue sections obtained from patients with breast cancer, most notably in sections from patients with basal-like breast cancer, where gal-7 is preferentially expressed [6]. The expression of gal-7 in cytosolic and nuclear compartments was also confirmed

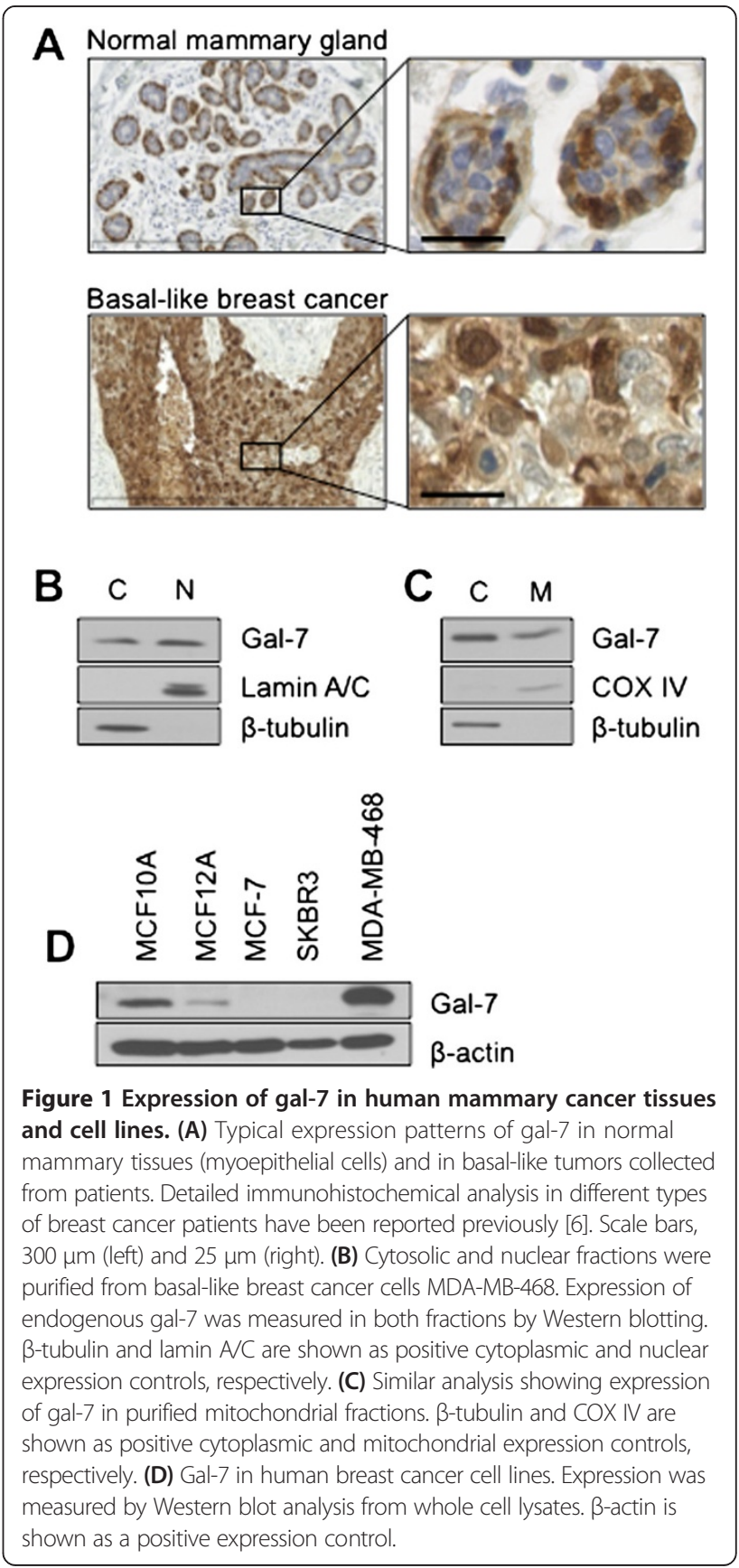


by western blot analysis of subcellular fractions isolated from MDA-MB-468 cells, which constitutively express gal-7 (Figure 1B-D). We also confirmed the ability of gal-7 to translocate to mitochondria in breast cancer cells, as recently observed in human colon carcinoma cells [11].

\section{Generation of the R74S mutant}

Site-directed mutagenesis was used to generate mutants of gal-7. A special attention was paid to arginine 74, the structural equivalent of arginine 186 in human galectin3. Mutation R186S was previously shown to abolish the biological function of galectin-3 [18]. The replacement of arginine 74 to serine (R74S) was thus introduced in the human gal-7 gene by oligo-directed site-specific mutagenesis. To verify the integrity and structural perturbations caused by the R74S mutation, we used solution NMR spectroscopy, which provides a fast and highly sensitive assessment of structural perturbations caused by point mutations in proteins. For this purpose, the wild-type gal-7 (wtgal-7) and variant R74S were isotopically labeled, overexpressed in E. coli BL21(DE3) and purified to homogeneity. Their two-dimensional heteronuclear single quantum coherence spectra $\left({ }^{1} \mathrm{H}-{ }^{15} \mathrm{~N}\right.$ HSQC) were then acquired and overlaid (Figure 2A). Our ${ }^{1} \mathrm{H}-^{15} \mathrm{~N}$ HSQC spectral analysis showed that the R74S mutation induced only limited and local changes to the gal-7 fold (Figure 2A-D). Recombinant forms of R74S and wtgal-7 were also equally effective at forming dimers in solution (Figure 2E). Analysis of the thermodynamic parameters of the proteins by isothermal titration calorimetry (ITC) indicated, however, that binding of lactose to gal-7 was partially inhibited by the R74S mutation, with a $K_{\mathrm{d}}$ value of $720 \mu \mathrm{M}$ for the R74S variant relative to $378 \mu \mathrm{M}$ for the wild-type protein. A typical titration profile is shown in Additional file 1: Figure S1. This finding was corroborated by our flow cytometric analysis showing that binding of recombinant R74S to glycan receptors on the surface of Jurkat $\mathrm{T}$ cells was significantly lower than that observed with the wild-type protein (Additional file 2: Figure S2).

\section{Functional characterization of the R74S mutant}

Because MCF-7 cells have been extensively used as a model system for human breast cancer, we have used this cell line to express and further characterize the R74S mutant. The relevance of MCF-7 model for our studies was first established by expressing the wild-type form of gal-7 (wtgal-7). Western blotting analysis of stable transfectants showed that wtgal-7 was present in cytosolic, nuclear, and mitochondrial extracts of MCF-7 cells transfected with an expression vector encoding wtgal-7 (Figure 3A-B), a pattern similar to that found in MDA-MB-468 cells. Immunogold immunohistochemistry by electron microscopy (EM) confirmed the presence of gal-7 in these subcellular compartments (Figure 3C-F). The gold beads labeled mitochondria on the outer membrane and inside the organelle. In all cases, gal-7 was expressed in clusters, mostly being found inside the
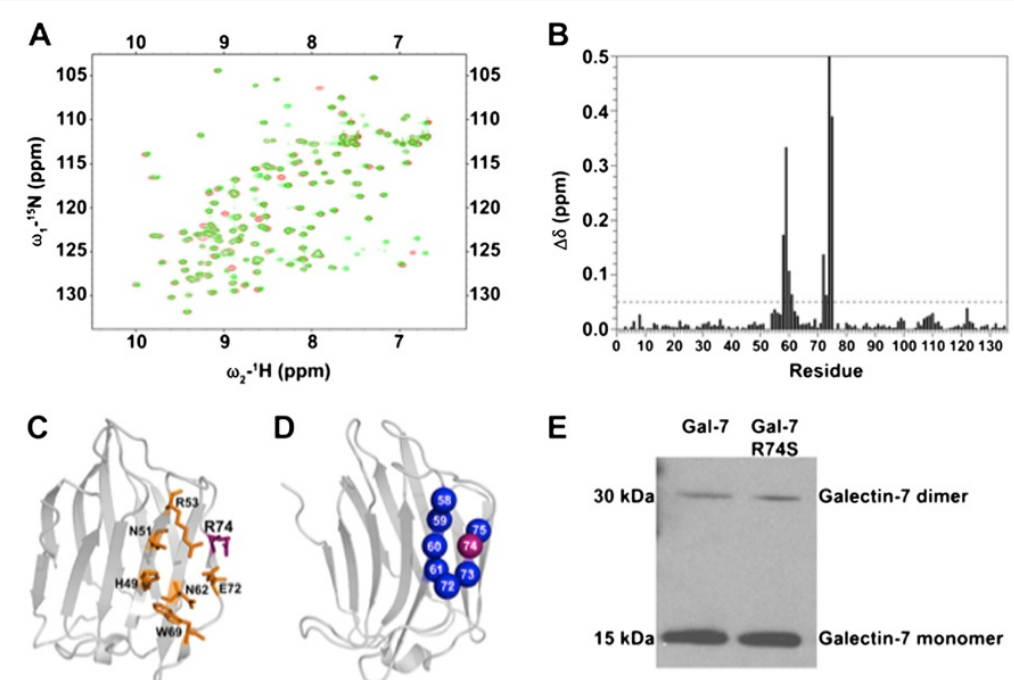

Figure 2 Structural analysis of wild-type gal-7 and the R74S mutant. (A) Superimposed ${ }^{1} \mathrm{H}^{-15} \mathrm{~N}$ HSQC spectra of wild-type (green) and R74S (red) gal-7 at $310 \mathrm{~K}$ and $800 \mathrm{MHz}$. (B) ${ }^{1} \mathrm{H}^{-15} \mathrm{~N}$ chemical shift differences $\Delta \delta$ (ppm) caused by the R74S mutation mapped on the primary sequence of gal-7. The ${ }^{1} \mathrm{H}_{-}{ }^{15} \mathrm{~N}$ weighted average composite chemical shift differences $(\Delta \delta)$ were calculated between $\mathrm{WT}$ and variant R74S according to the following equation [17]: $\Delta \delta(\mathrm{ppm})=\left[\left(\Delta \delta_{\mathrm{HN}}^{2}+\Delta \delta_{N}^{2} / 25\right) / 2\right]^{1 / 2}$. (C) Three-dimensional structure of gal-7 showing the general $\beta$-sheet topology of the carbohydrate recognition domain (CRD) and the position of the carbohydrate binding site, as delineated by residues H49, N51, R53, N62, W69, E72 and R74. (D) Mapping of ${ }^{1} \mathrm{H}^{15} \mathrm{~N}$ chemical shift variations $(\Delta \delta)$ between wtgal-7 and variant R74S on the 3D structure of gal-7 [PDB: 3ZXF]. Residues with chemical shift variations $\Delta \delta>0.05 \mathrm{ppm}$ are plotted on the structure of gal-7 (in blue). The position of the R74 residue is shown in purple. (E) Immunoblots showing soluble monomeric and dimeric forms of recombinant gal-7 in a native gel. 


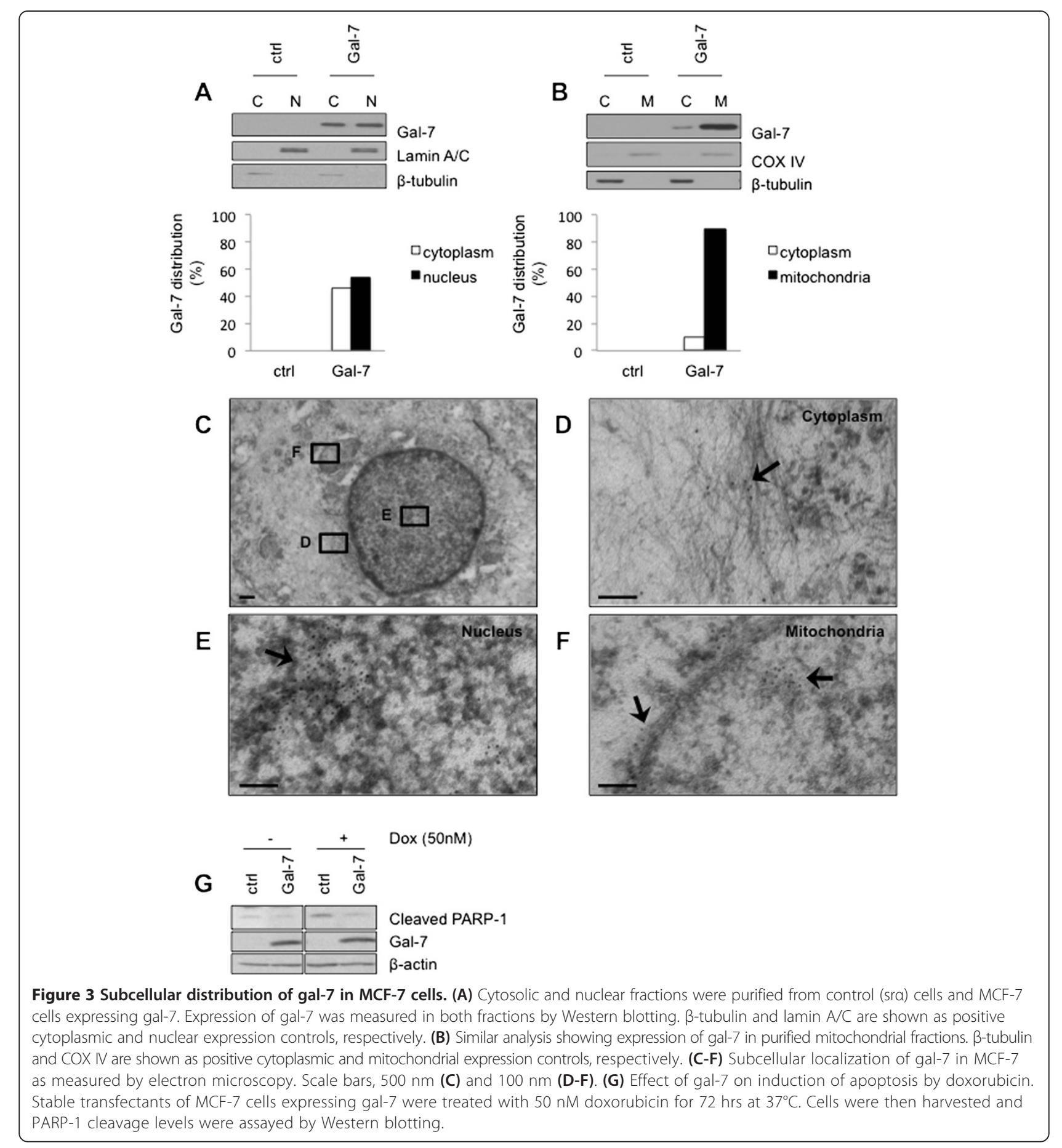

mitochondria (Additional file 3: Figure S3). MCF-7 expressing wtgal-7 was also more resistant to apoptosis induced by doxorubicin (dox) as compared to control MCF-7 cells transfected with a (empty) control vector (Figure 3G). These results corroborated our previous data using the mouse $4 \mathrm{~T} 1$ breast cancer cells [6]. We thus examined whether the $\mathrm{R} \rightarrow \mathrm{S}$ mutation at position 74 induced a change in the subcellular localization of gal-7. Our Western blot analyses showed that this mutation greatly reduced the expression of gal-7 in the nucleus and mitochondria (Figure 4A-B). This effect was confirmed by EM studies (Figure 4C-F). Interestingly, however, we found that MCF-7 cells expressing wild-type and R74S forms of gal-7 were equally resistant to apoptosis induced by cobalt chloride, a hypoxia mimicking agent (Figure 5A-B), and by other anticancer drugs, 


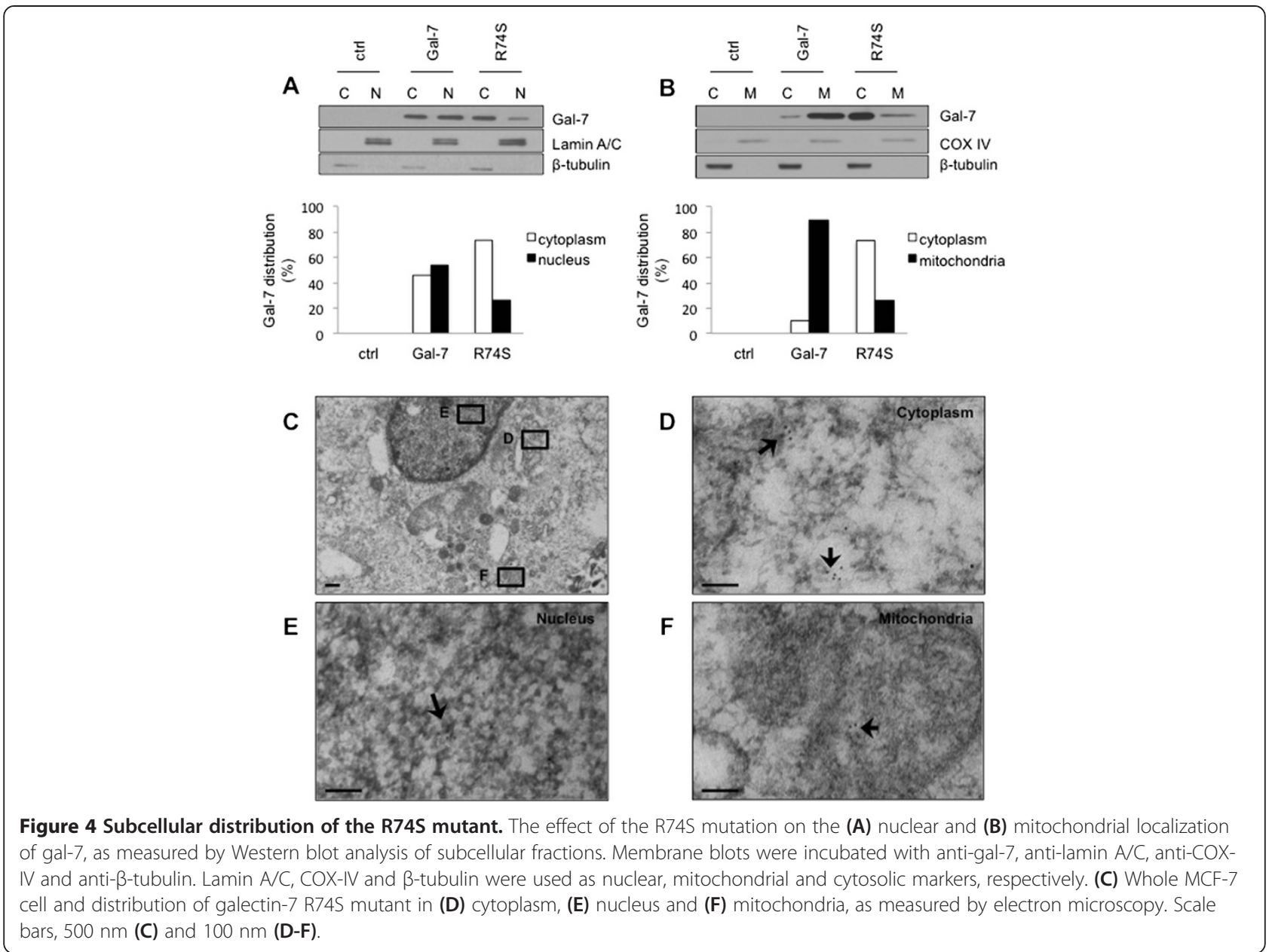

such as etoposide and doxorubicin (dox) (Figures 5C and $6 \mathrm{~A})$. In fact, MCF-7 cells expressing R74S were more resistant to apoptosis induced by etoposide and dox.

\section{Gal-7 reduces p53-induced p21 expression}

Because MCF-7 cells express a wild-type form of p53 and that DNA damage response induced by dox is known to increase the cyclin-independent kinase inhibitor p21 via a p53-dependent pathway, we took this opportunity to examine the expression of p21 and p53 in MCF-7 cells expressing wtgal-7 and R74S. We found that both wtgal-7 and R74S inhibited dox-induced PARP-1 cleavage and p53 protein expression (Figure 6A-B). The inhibition of p53 correlated with a decrease in $\mathrm{p} 21$ protein expression and CDKN1A mRNA (Figure 6B-C). Again, the inhibition by R74S was stronger than that observed with the wild-type form of gal-7. Furthermore, analysis of nuclear and cytoplasmic fractions showed that wild type and R74S mutant gal-7 inhibited p53 nuclear translocation (Figure 7A). Treatment of cells with MG-132, a well-known proteasome inhibitor, restored the p53 expression, suggesting that both forms of gal-7 promote degradation of cytosolic p53 (Figure 7B). This possibility is supported by our data showing that both forms of gal-7 co-precipitate with p53 (Figure 7C-D). The ability of endogenous gal7 to co-precipitate with p53 was further confirmed using MCF10A cells (Additional file 4: Figure S4).

\section{Discussion}

There is an increasing interest in the development of galectin-specific inhibitors for the treatment of cancer. Because galectins exert both intracellular and extracellular functions, a better understanding of their subcellular localization in cancer cells is critical to promote the development of new anti-cancer therapies directed at these proteins.

Gal-7 is highly expressed at both the mRNA and protein levels in tissues of patients with aggressive forms of cancer, including basal-like breast cancer subtype $[6,19,20]$. Experimentally, gal-7 has been shown to increase the metastatic behavior of cancer cells while its suppression reduces their metastatic behavior [6,21,22]. Like other members of the galectin family, however, gal-7 has been shown to have a dual role in cancer. While it promotes 


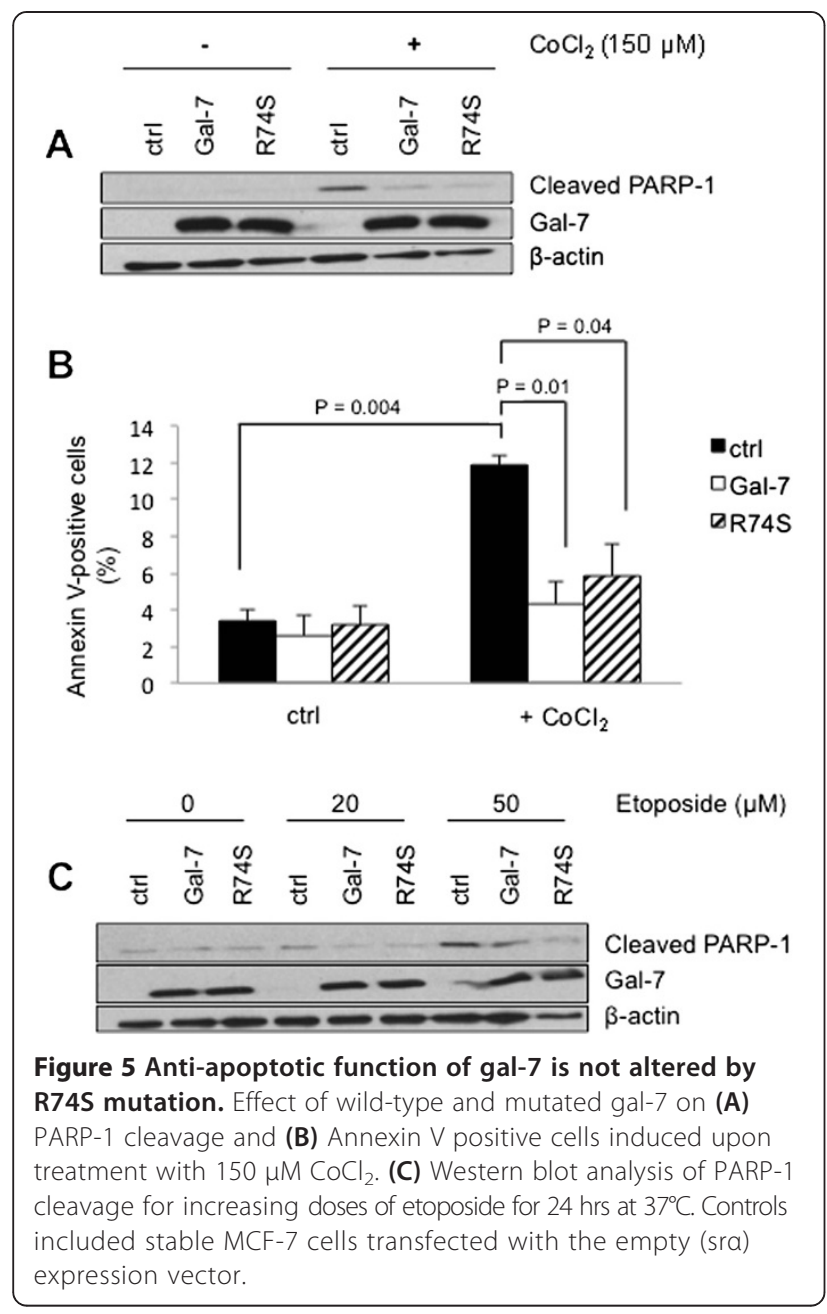

cancer progression in many types of cancer, it may exert an anti-tumor activity in other types of cancer, such as urothelial carcinoma and colon cancer. In all cases, the role of gal-7 in apoptosis was associated with its intracellular localization, as shown by the strong cytosolic and nuclear immunoreactivity with anti-gal-7 antibodies [6,23]. Our model system with the R74S mutant will thus be useful to determine whether translocation to mitochondria and nucleus modulates the ability of gal-7 to modulate apoptosis in other cancer cell types.

Because R74 is located in the vicinity of the CRD, it is not surprising that a mutation at this position reduces the affinity to lactose or the binding to cell surface glycoproteins. It may also affect the fine specificities of ligand recognition in the ligand binding groove, as suggested by the tridimensional structure of gal-7 [4]. We expect, however, that the R74S mutation will not affect the proteinprotein interactions that gal-7 displays with proteins such as Bcl-2 and Smad3 $[9,11]$. Similarly to galectin-3 that utilizes synexin for its translocation to the perinuclear mitochondrial membranes, gal-7 might also require the aid of

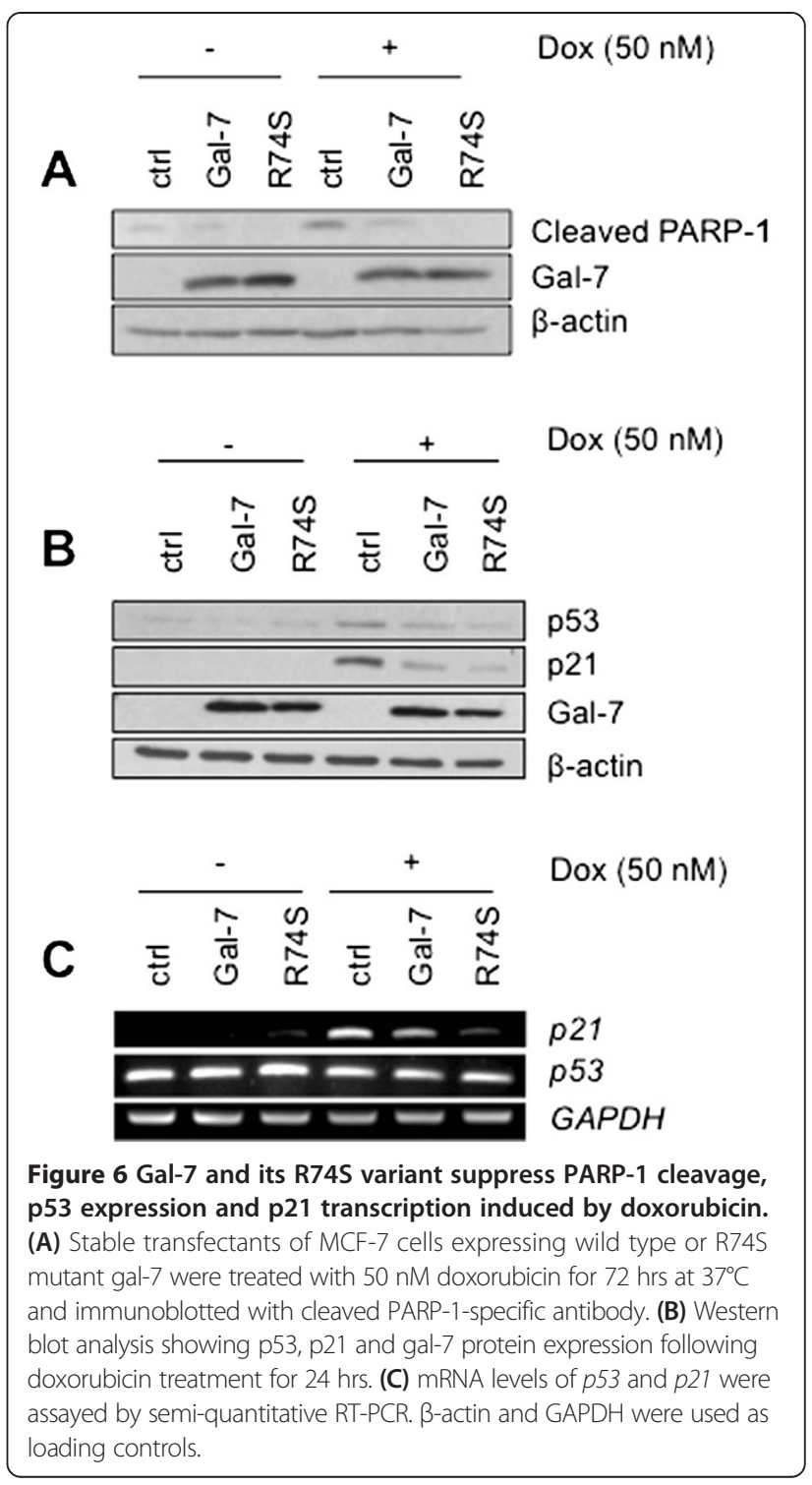

similar transport proteins for its translocation [24]. As such, the cytosolic presence of the R74S mutant is potentially due to the loss of the interaction between gal-7 and its transport proteins resulting in its pronounced cytosolic localization. Alternatively, the mutation at the R74 promoting the cytosolic sequestration of gal-7 may allow enhanced binding to cytosolic proteins increasing as such various cytoplasmic signaling pathways. Nevertheless, future studies will be needed to determine the specific mechanism by which gal-7 translocates to mitochondria and to the nucleus.

Our results suggest that gal-7 may be involved in the regulation of p21 expression. These results may thus provide a new mechanism underlying the functions of gal-7 in apoptosis and warrant further investigation. Specifically, we found that the R74S mutation does not alter the proliferation rate of breast cancer cells (Additional file 5: Figure S5). 


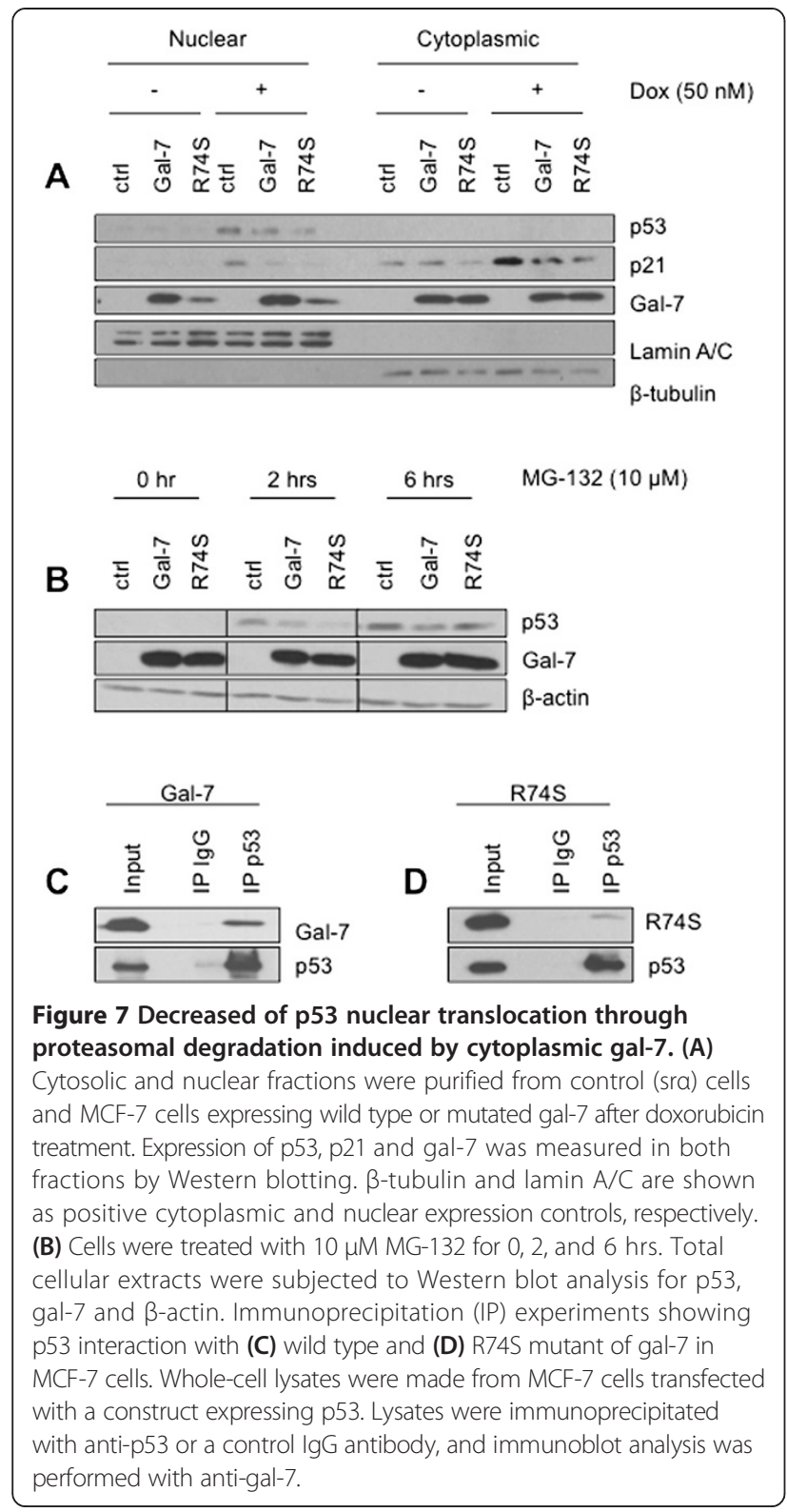

Rather, our data obtained using the proteasome inhibitor and the co-immunoprecipitation of gal-7 with p53 suggests that gal-7 may help to stabilize cytosolic p53, possibly by modulating its interaction with MDM2. Whether gal-7 directly binds to p53 or belongs to the p53 multimolecular complex is currently unknown. Although glycosylation of p53 has been reported [25-27] and that some p53interacting proteins are glycosylated [28], our observation that R74S also co-immunoprecipitates with p53 suggests that such interaction could be CRD-independent. Such CRD-independent function for galectins is not uncommon, especially for intracellular galectins [3]. Another possibility that may explain lower levels of nuclear p53 protein and reduced $\mathrm{p} 21$ activation is that gal-7 may be part of a complex network of interrelated mechanisms that regulate the nucleo-cytoplasmic transport of p53 following cellular stress. These possibilities are currently under investigation.

\section{Conclusions}

In the present work, we have shown that: 1) a mutation at position 74 inhibited translocation of gal-7 to the mitochondria and the nucleus, sequestering gal-7 to the cytosolic compartment; 2) such decrease of gal-7 expression in the nucleus and mitochondria does not impair the ability of gal-7 to drug-induced apoptosis; in fact, the R74S mutant protected even more cells from apoptosis induced by anti-cancer drugs, and 3) sequestration of gal-7 to the cytosol impaired the translocation of p53 to the nucleus and the upregulation of p21. Taken together, these results suggest that targeting cytosolic gal-7 in breast cancer cells may be a valuable strategy for the treatment of this disease.

\section{Additional files}

Additional file 1: Figure S1. Isothermal calorimetric titration of gal-7 with lactose. (Top) Typical ITC experiment carried out by adding $2 \mu$ laliquots of $6 \mathrm{mM}$ lactose to $200 \mu \mathrm{M}$ wtgal-7 or R74S. (Bottom) Heat released per mole of lactose injected as a function of the sugar / protein molar ratio. The titration was obtained at $25^{\circ} \mathrm{C}$ in $20 \mathrm{mM}$ potassium phosphate buffer, $\mathrm{pH} 7.2$.

Additional file 2: Figure S2. Binding of recombinant wtgal-7 and R74S to Jurkat T cells. Increasing concentrations of recombinant FITC-labeled wtgal-7 or R74S were added to Jurkat T cells. Binding was measured by flow cytometry after $30 \mathrm{~min}$ of incubation at $4^{\circ} \mathrm{C}$.

Additional file 3: Figure S3. Clusters of gal-7 in MCF-7 cells observed by electron microscopy. Transmission electron micrographs showing gal-7 clusters (arrows) in (A) the cytoplasm, (B) nucleus, and (C) mitochondria of MCF-7 stable transfectants expressing gal-7. In (D), quantitative assessment of the number of immunogold particles per clusters as measured from transmission electron micrographs.

Additional file 4: Figure S4. Interaction of endogenous gal-7 with p53 in MCF10A cells. Immunoprecipitation (IP) experiments showing p53 interaction with endogenous gal-7 in MCF10A cells. Whole-cell lysates were made from MCF10A cells transfected with a construct expressing p53. Lysates were IP with anti-p53 or a control IgG antibody, and immunoblot analysis was performed with anti-gal-7.

Additional file 5: Figure S5. Cellular proliferation of MCF-7 is not significantly affected by gal-7 and R74S variant. Stable transfectants of MCF-7 cells expressing empty vector (ctrl), wild type or R74S mutant gal-7 were seeded at 5000 cells per well in E-Plates 96 and observed for a period of $72 \mathrm{~h}$. Dynamic proliferation assay was achieved via xCELLigence real-time cell analyzer.

\section{Abbreviations}

gal-7: Galectin-7; wtgal-7: Wild-type galectin-7; CRD: Carbohydrate recognition domain; dox: Doxorubicin.

\section{Competing interests}

The authors declare that they have no competing interests.

\section{Authors' contributions}

Conceived and designed the experiments: AAG, YSP, ND and LG. Performed the experiments and analyzed the data: AAG, ML, MCV and DG. Wrote the paper: AAG and YSP. All authors read and approved the final manuscript. 


\section{Acknowledgements}

The authors thank Diane Tremblay, Isabelle Plante, and Micheline Letarte (INRS) for their excellent technical support, as well as Sameer Al-Abdul-Wahid from the Québec/Eastern Canada High Field NMR Facility for his excellent NMR technical assistance.

Supported in part by grants to Y.S-P. from the Canadian Institute for Health Research (Grant No. MOP-89697) and a Discovery grant to N.D. from the Natural Sciences and Engineering Research Council of Canada (RGPIN 402623-2011). A.A.G. and M.L. are supported by a Ph.D. studentship and N.D. by a Research Scholar Career Award (Junior 1) from the Fonds de Recherche du Québec-Santé (FRQS). D.G. is supported by a Ph.D. studentship from the Fondation universitaire Armand-Frappier de I'INRS. N.D. also acknowledges support from the FRQNT Strategic Cluster Regroupement Québécois de Recherche sur la Fonction, la Structure et I'Ingénierie des Protéines (PROTEO) and the FRQS Strategic Cluster Groupe de Recherche Axé sur la Structure des Protéines (GRASP).

\section{Author details}

'INRS-Institut Armand-Frappier, 531 Blv. des Prairies, Laval, Quebec H7V 1B7, Canada. ${ }^{2}$ RIC | Université de Montréal, 2950 Chemin de Polytechnique, Montreal, Quebec H3T 1J4, Canada.

Received: 1 July 2014 Accepted: 9 October 2014

Published: 3 November 2014

\section{References}

1. Di Lella S, Sundblad V, Cerliani JP, Guardia CM, Estrin DA, Vasta GR, Rabinovich GA: When galectins recognize glycans: from biochemistry to physiology and back again. Biochemistry 2011, 50:7842-7857.

2. Brewer CF, Miceli MC, Baum LG: Clusters, bundles, arrays and lattices: novel mechanisms for lectin-saccharide-mediated cellular interactions. Curr Opin Struct Biol 2002, 12:616-623.

3. Vladoiu MC, Labrie M, St-Pierre Y: Intracellular galectins in cancer cells: potential new targets for therapy (Review). Int J Oncol 2014, 44:1001-1014.

4. Leonidas DD, Vatzaki EH, Vorum H, Celis JE, Madsen P, Acharya KR Structural basis for the recognition of carbohydrates by human galectin-7. Biochemistry 1998, 37:13930-13940.

5. Magnaldo T, Fowlis D, Darmon M: Galectin-7, a marker of all types of stratified epithelia. Differentiation 1998, 63:159-168.

6. Demers M, Rose AA, Grosset AA, Biron-Pain K, Gaboury L, Siegel PM, St-Pierre Y: Overexpression of galectin-7, a myoepithelial cell marker, enhances spontaneous metastasis of breast cancer cells. Am J Pathol 2010, 176:3023-3031.

7. St-Pierre Y, Campion CG, Grosset AA: A distinctive role for galectin-7 in cancer? Front Biosci 2010, 17:438-450

8. Campion CG, Labrie M, Lavoie G, St-Pierre Y: Expression of galectin-7 is induced in breast cancer cells by mutant p53. PLOS One 2013, 8:e72468.

9. Inagaki Y, Higashi K, Kushida M, Hong YY, Nakao S, Higashiyama R, Moro T, Itoh J, Mikami T, Kimura T, Shiota G, Kuwabara I, Okazaki I: Hepatocyte growth factor suppresses profibrogenic signal transduction via nuclear export of Smad3 with galectin-7. Gastroenterology 2008, 134:1180-1190.

10. Cada Z, Chovanec M, Smetana K, Betka J, Lacina L, Plzak J, Kodet R, Stork J, Lensch M, Kaltner H, Andre S, Gabius HJ: Galectin-7: will the lectin's activity establish clinical correlations in head and neck squamous cell and basal cell carcinomas? Histol Histopathol 2009, 24:41-48.

11. Villeneuve C, Baricault L, Canelle L, Barboule N, Racca C, Monsarrat B, Magnaldo T, Larminat F: Mitochondrial proteomic approach reveals galectin-7 as a novel BCL-2 binding protein in human cells. Mol Biol Cell 2011, 22:999-1013.

12. Vander Ghinst M, Remmelink M, Mansbach AL, Hassid S, Choufani G: Galectin-1, $-3,-7$ expressions in congenital and acquired pediatric cholesteatomas compared to external auditory canal skin. Clin Exp Otorhinolaryngol 2012, 5:62-67.

13. Doucet N, Watt ED, Loria JP: The flexibility of a distant loop modulates active site motion and product release in ribonuclease A. Biochemistry 2009, 48:7160-7168.

14. Morris S, Ahmad N, Andre S, Kaltner H, Gabius HJ, Brenowitz M, Brewer F: Quaternary solution structures of galectins-1, -3 , and -7 . Glycobiology 2004, 14:293-300.
15. Delaglio F, Grzesiek S, Vuister GW, Zhu G, Pfeifer J, Bax A: NMRPipe: a multidimensional spectral processing system based on UNIX pipes. J Biomol NMR 1995, 6:277-293.

16. Kneller D, Kuntz I: UCSF Sparky: an NMR display. Annotation and Assignment Tool, University of California, San Francisco 1993, 254:.

17. Grzesiek S, Stahl SJ, Wingfield PT, Bax A: The CD4 determinant for downregulation by HIV-1 Nef directly binds to Nef. Mapping of the Nef binding surface by NMR. Biochemistry 1996, 35:10256-10261.

18. Salomonsson E, Carlsson MC, Osla V, Hendus-Altenburger R, Kahl-Knutson B, Oberg CT, Sundin A, Nilsson R, Nordberg-Karlsson E, Nilsson UJ, Karlsson A, Rini JM, Leffler H: Mutational tuning of galectin-3 specificity and biological function. J Biol Chem 2010, 285:35079-35091.

19. Chung $\mathrm{CH}$, Bernard PS, Perou CM: Molecular portraits and the family tree of cancer. Nat Genet 2002, 32(Suppl):533-540.

20. Jones C, Mackay A, Grigoriadis A, Cossu A, Reis-Filho JS, Fulford L, Dexter T, Davies S, Bulmer K, Ford E, Parry S, Budroni M, Palmieri G, Neville AM, O'Hare MJ, Lakhani SR: Expression profiling of purified normal human luminal and myoepithelial breast cells: identification of novel prognostic markers for breast cancer. Cancer Res 2004, 64:3037-3045.

21. Demers M, Magnaldo T, St-Pierre Y: A novel function for galectin-7: promoting tumorigenesis by up-regulating MMP-9 gene expression. Cancer Res 2005, 65:5205-5210.

22. Demers M, Biron-Pain K, Hebert J, Lamarre A, Magnaldo T, St-Pierre Y: Galectin-7 in lymphoma: elevated expression in human lymphoid malignancies and decreased lymphoma dissemination by antisense strategies in experimental model. Cancer Res 2007, 67:2824-2829.

23. Kuwabara I, Kuwabara Y, Yang RY, Schuler M, Green DR, Zuraw BL, Hsu DK, Liu FT: Galectin-7 (PIG1) exhibits pro-apoptotic function through JNK activation and mitochondrial cytochrome c release. J Biol Chem 2002, 277:3487-3497.

24. Yu F, Finley RL Jr, Raz A, Kim HR: Galectin-3 translocates to the perinuclear membranes and inhibits cytochrome $\mathrm{c}$ release from the mitochondria. A role for synexin in galectin-3 translocation. J Biol Chem 2002, 277:15819-15827.

25. Shaw P, Freeman J, Bovey R, Iggo R: Regulation of specific DNA binding by $\mathrm{p} 53$ : evidence for a role for O-glycosylation and charged residues at the carboxy-terminus. Oncogene 1996, 12:921-930.

26. Bode AM, Dong Z: Post-translational modification of $\mathrm{p} 53$ in tumorigenesis. Nat Rev Cancer 2004, 4:793-805.

27. Yang WH, Kim JE, Nam HW, Ju JW, Kim HS, Kim YS, Cho JW: Modification of p53 with O-linked $\mathrm{N}$-acetylglucosamine regulates p53 activity and stability. Nat Cell Biol 2006, 8:1074-1083.

28. Lui K, An J, Montalbano J, Shi J, Corcoran C, He Q, Sun H, Sheikh MS, Huang Y: Negative regulation of $\mathrm{p} 53$ by Ras superfamily protein RBEL1A. J Cell Sci 2013, 126:2436-2445.

doi:10.1186/1471-2407-14-801

Cite this article as: Grosset et al: Cytosolic galectin-7 impairs p53 functions and induces chemoresistance in breast cancer cells. BMC Cancer 2014 14:801.

\section{Submit your next manuscript to BioMed Central and take full advantage of:}

- Convenient online submission

- Thorough peer review

- No space constraints or color figure charges

- Immediate publication on acceptance

- Inclusion in PubMed, CAS, Scopus and Google Scholar

- Research which is freely available for redistribution 